\title{
Flt3/Flt3L Participates in the Process of Regulating Dendritic Cells and Regulatory T Cells in DSS-Induced Colitis
}

\author{
Jing-Wei Mao, ${ }^{1}$ Yu-Shuang Huang, ${ }^{2}$ Hai-Ying Tang, ${ }^{1}$ Jian Bi, \\ Yan-Fei Liu, ${ }^{1}$ and Ying-De Wang ${ }^{1}$ \\ ${ }^{1}$ Department of Gastroenterology, the First Affiliated Hospital of Dalian Medical University, 222 Zhongshan Road, \\ Dalian, Liaoning 116011, China \\ ${ }^{2}$ Department of Liver Diseases, Dalian Sixth People’s Hospital, 269 Guibai Road, Dalian, Liaoning 116037, China
}

Correspondence should be addressed to Ying-De Wang; albertwyd@163.com

Received 22 May 2014; Revised 4 August 2014; Accepted 27 August 2014; Published 13 October 2014

Academic Editor: Gerassimos Mantzaris

Copyright (C) 2014 Jing-Wei Mao et al. This is an open access article distributed under the Creative Commons Attribution License, which permits unrestricted use, distribution, and reproduction in any medium, provided the original work is properly cited.

\begin{abstract}
The immunoregulation between dendritic cells (DCs) and regulatory T cells (T-regs) plays an important role in the pathogenesis of ulcerative colitis (UC). Recent research showed that Fms-like tyrosine kinase 3 (Flt3) and Flt3 ligand (Flt3L) were involved in the process of DCs regulating T-regs. The DSS-induced colitis model is widely used because of its simplicity and many similarities with human UC. In this study, we observe the disease activity index (DAI) and histological scoring, detect the amounts of DCs and T-regs and expression of Flt3/Flt3L, and investigate Flt3/Flt3L participating in the process of DCs regulating T-regs in DSS-induced colitis. Our findings suggest that the reduction of Flt3 and Flt3L expression may possibly induce colonic immunoregulatory imbalance between $\mathrm{CD}_{103}{ }^{+} \mathrm{MHCII}^{+} \mathrm{DCs}$ and $\mathrm{CD} 4^{+} \mathrm{CD} 25^{+} \mathrm{FoxP}^{+}$T-regs in DSS-induced colitis. Flt3/Flt3L participates in the process of regulating DCS and T-regs in the pathogenesis of UC, at least, in the acute stage of this disease.
\end{abstract}

\section{Introduction}

UC is a nonspecific inflammatory bowel disease (IBD) for which the etiology and pathogenesis are still not completely clear so far. One of the hypotheses regarding its pathogenesis involves the dysfunction of intestinal mucosal homeostasis due to a genetically determined miscommunication between commensal flora and intestinal immune system [1].

DCs are pivotal in tolerance induction and direct the differentiation of $\mathrm{T}$ cells [2]. Microbial antigen handled by DCs is believed to be of critical importance for immunity and tolerance in UC. Mucosal DCs play an important role in the development of oral tolerance, a phenomenon in which immune responses to a defined protein are blunted when the same protein has been orally fed before the rodents were systemically challenged [3]. The triggering of oral tolerance in patients with UC is impaired [4]. Interactions between DCs and $\mathrm{T}$ cells seem to be essential for the development of oral tolerance, in which $\mathrm{CD} 4^{+} \mathrm{CD} 25^{+} \mathrm{FoxP}^{+} \mathrm{T}$-regs that suppress systemic immune responses are primed [5]. T-regs have an essential role in maintaining immune tolerance in the gut and in the control of immune pathology in UC.
Recent studies demonstrated that there had been crosstalk between DCs and T-regs. Meanwhile, Flt3 and Flt3L were involved in this process $[6,7]$. Flt3, a member of the tyrosine-kinase receptor family, was initially cloned from fetal liver cells with hematopoietic stem cell activity. Flt3L is the ligand for Flt3, which is a key regulatory cytokine for DCs commitment and development.

So far, very few studies have been performed regarding the participation of Flt3/Flt3L in the regulation of DCs and Tregs in UC. In this study, we aimed to clarify the role of Flt3/ Flt3L in the immunoregulation between DCs and T-regs by detecting the colonic expression of Flt3/Flt3L and quantity of DCs and T-regs in terms of percentage in DSS-induced acute colitis.

\section{Materials and Methods}

2.1. Materials. Healthy male BALB/c mice weighing about $25 \mathrm{~g}$ were supplied by the Specific Pathogen Free (SPF) Laboratory Animal Center of Dalian Medical University. DSS was 
purchased from Sigma. FITC-conjugated anti-mouse CD103, PE-conjugated anti-mouse I-A/I-E (MHC-II), PE-conjugated anti-mouse CD4, PerCP-conjugated anti-mouse CD25, and PE-conjugated anti-mouse FOXP3 were supplied by BioLegend Company. Flt3 polyclonal antibody was supplied by Santa Company. The MaxVision TM plus Poly HRP (Mouse/ Rabbit) IHC Kit was supplied by Beijing Zhongshan Goldenbridge Biotechnology Co., Ltd. Primers, PrimeScript RT Master Mix Perfect Real-Time kit, SYBR Premix Ex Taq (Tli RNaseH Plus), and DEPC were from Dalian, Takara Co., Ltd. ELISA kit for Flt3L was purchased from BIOVALUE Company.

2.2. Animals. A total of $20 \mathrm{BALB} / \mathrm{c}$ mice were randomly divided into two groups with 10 animals in each, the control group and DSS-induced colitis model group. Mice in the control group were fed with general diet and distilled water. The mice in model group were induced by oral administration of $50 \mathrm{~g} / \mathrm{L}$ dextran sulphate sodium (DSS) solution every day for 7 days. Mice from two groups were sacrificed on day 7 and colonic tissue was collected for flow cytometry method (FCM), Real-time PCR, HE staining, and immunohistochemistry analysis. The blood samples of $0.8 \mathrm{~mL}$ from the heart were obtained for ELISA.

Mice were kept in a normally controlled breeding room with standard laboratory food and water for one week before the experiments. All experimental procedures were conducted according to the institutional guidelines for the care and use of Laboratory Animals of Dalian Medical University and conformed to the National Institutes of Health Guide for Care and Use of Laboratory Animals (Publication no. 85-23, revised in 1996). All protocols were approved by the Institutional Animal Care and Use Committee of Dalian Medical University.

2.3. Determination of DAI. Mice were weighed and checked for stool consistency and the presence of gross blood in the stool every day during the experiment. The DAI scores were assigned as follows: percentage of body weight reduction (0: no change; 1 : $1-5 \% ; 2$ : 6-10\%; 3: $11-15 \%$; 4 : >15\%); stool consistency ( 0 : normal; 2 : loose; 4 : diarrhea); and the presence of fecal blood (0: normal; 2: positive occult blood test; 4: visible bleeding) [8]. The DAI was calculated as the sum of these scores divided by 3 .

2.4. Determination of Histological Scoring. After mice were sacrificed, the entire colon was removed for gross colonic pathological damage observation. The colon samples with the worst damages were formalin-fixed, paraffin-embedded, and cut into $4 \mu \mathrm{m}$ thick sections for HE staining and histological analysis. Slides were examined and scored in a blinded fashion using the published grading system [9]. The scores were measured as follows: severity of inflammation (0: none; 1: slight; 2 : moderate; and 3: severe), depth of injury (0: none; 1: mucosal; 2: mucosal and submucosal; and 3: transmural), and crypt damage (0: none; 1 : basal one-third damaged; 2 : basal two-thirds damaged; 3: only surface epithelium intact; and 4: entire crypt and epithelium lost).
2.5. Detecting DCs and T-Regs by FCM. The fresh clean colonic tissues about $0.2 \mathrm{~g}$ were mechanically disaggregated into a single cell suspension by using $75 \mu \mathrm{m}$ mesh. PBS-BSAETDA solution was added to the cells for a concentration of $1 \times 10^{6} / \mathrm{mL}$. The single cell suspension was centrifuged for $5 \mathrm{~min}$ at $1500 \times \mathrm{g}$ at room temperature. After removing the supernatant, cells were incubated with $5 \mu \mathrm{L}$ PE-conjugated anti-mouse I-A/I-E and FITC-conjugated anti-mouse CD103 for $30 \mathrm{~min}$ at $4^{\circ} \mathrm{C}$. The cells were then washed, centrifuged, and resuspended in $0.5 \mathrm{~mL}$ PBS before subjecting them to FCM for detecting DCs. For detecting T-regs, cells were stained with PE-conjugated anti-mouse CD4 and PerCPconjugated anti-mouse CD25 for 30 minutes at $4^{\circ} \mathrm{C}$. Cells were subsequently washed in staining buffer twice prior to fixation and permeabilization using eBioscience's buffers for intracellular staining of FoxP3 according to the manufacturer's instructions. The cells were then washed, centrifuged, and resuspended in $0.5 \mathrm{~mL}$ PBS before subjecting them to FCM.

2.6. Flt3 and Flt3L mRNA Expression by Real-Time PCR. Total RNA was extracted from colonic tissue samples using Trizol according to the manufacturer's protocols (Invitrogen). All RNA samples were checked for RNA quality by gel electrophoresis. Real-time PCR was performed by using SYBR Green I as a double-strand DNA-specific binding dye and continuous fluorescence monitoring. Reverse transcription (RT) reactions were performed using $0.5 \mu \mathrm{L}$ total RNA, $2 \mu \mathrm{L}$ PrimeScript RT Master Mix, and $7.5 \mu \mathrm{L}$ RNase Free $\mathrm{dH}_{2} \mathrm{O}$. Amplification was performed in a total volume of $20 \mu \mathrm{L}$ containing $0.4 \mu \mathrm{L}$ of PCR forward primer and reverse primer, $0.4 \mu \mathrm{L}$ of ROX reference dye II, $10 \mu \mathrm{L}$ of SYBR Premix Ex Taq, $6.8 \mu \mathrm{L}$ of $\mathrm{dH}_{2} \mathrm{O}$, and $2.0 \mu \mathrm{L}$ of DNA. An equal amount of cDNA from each sample was amplified using primers specific to each gene. The qPCR primer sequences for Flt3 were Flt3-F: GGTTTAAAGCGTACCCACGA and Flt3-R: CTCCAGGCCTCTCGTTCAC; for Flt3L were Flt3L-F: GAGGACGTCAACACCGAGAT and Flt3L-R: AGGTGGGAGATGTTGGTCTG; for GAPDH were GAPDH-F: CGTCCCGTAGACAAAATGGT and GAPDHR: TTGATGGCAACCAATCTCCAC. The PCR reactions were done under the following conditions: 40 cycles of initial denaturation at $95^{\circ} \mathrm{C}$ for $30 \mathrm{~s}$, denaturation at $95^{\circ} \mathrm{C}$ for $5 \mathrm{~s}$, annealing at $60^{\circ} \mathrm{C}$ for $34 \mathrm{~s}$, and extension at $72^{\circ} \mathrm{C}$ for $60 \mathrm{~s}$. Melting curve analysis of amplification products was performed at the end of each PCR reaction. All qRT-PCR assays were performed in triplicate.

2.7. Measurement of Flt3 Protein Expression in Colonic Mucosa and Flt3L in Plasma by Immunohistochemistry and ELISA. Immunohistochemistry for Flt3 colonic mucosal expression was done according to the MaxVision TM plus Poly HRP IHC Kit protocol. Image analysis software (Image-Pro Plus 6.0) was used to measure the light density of positive control cells in which the cytoplasm was tan-yellow or brown after $3,3^{\prime}$-diaminobenzidine (DAB) staining. For each section, the positive integrated optical density (IOD) and total area of five representative visual fields without overlap were observed under high-power microscope $(\times 400)$. The ratio of IOD and 

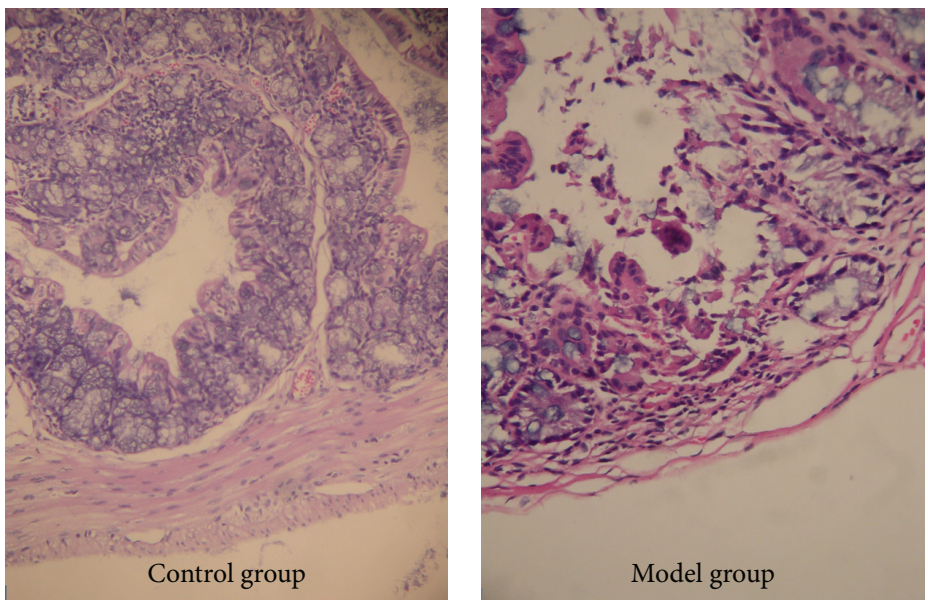

FIGURE 1: Pathological changes in the control group and UC model group on day 7 under light microscope (HE $\times 400)$. The colonic mucosa structure was intact in the control group. Mucosa and submucosa defects could be seen with infiltrations of inflammatory neutrophils and lymphocytes in the lamina propria in the UC model group.

total area represented the mean value of optical density, with a higher ratio indicating a higher level of protein expression.

Flt3L protein expression in plasma was measured utilizing Flt3L ELISA kit following the manufacturer's instructions. Absorbance was observed and recorded under $450 \mathrm{~nm}$ in 30 minutes after termination reaction by ELISA. The corresponding concentration was found according to the absorbance values on the standard curve. A higher concentration indicates a greater quantity of protein expression.

2.8. Statistical Analysis. Data analysis was performed using SPSS version 18.0 (SPSS, Chicago, IL, USA) statistical software. Data showed a normal distribution and were expressed as means \pm standard deviation. The results in different experimental groups were analyzed using one-way ANOVA. A $P$ value of $<0.05$ indicates a statistically significant difference between different groups.

\section{Results}

3.1. Results of DAI and Histological Scoring. In the control group, mice behavior, physical status, diets, and defecation were normal. On the contrary, mice in the model group showed different degrees of lethargy, poor diets, purulent blood stool, and weight loss.

Under light microscope, the colonic mucosa structure was intact in the control group. Mucosa and submucosa defects could be seen with infiltrations of inflammatory neutrophils and lymphocytes in the lamina propria in the model group (Figure 1).

The DAI and histological scoring in the model group were significantly higher than those in the control group (DAI: $3.15 \pm 0.28$ versus $0.00 \pm 0.00, P<0.05$; histological scoring: $7.62 \pm 0.40$ versus $0.00 \pm 0.00, P<0.05)$.

3.2. The Amount of $\mathrm{CD} 103^{+} \mathrm{MHCII}^{+} \mathrm{DCs}$ and $C D 4^{+} \mathrm{CD} 25^{+}$FoxP $3^{+}$T-Regs in Colonic Mucosa in terms of Percentage. In the model group and the control group, the percentage of $\mathrm{CD} 103^{+} \mathrm{MHCII}^{+} \mathrm{DC}$ of total cells was $(0.16 \pm$ $0.10) \%$ and $(2.24 \pm 1.03) \%$, respectively, and the percentage of $\mathrm{CD}^{+} \mathrm{CD}^{2} 5^{+} \mathrm{FoxP}^{+} \mathrm{T}$-regs in $\mathrm{CD} 4^{+} \mathrm{T}$ cells was $(4.11 \pm$ $2.14) \%$ and $(14.02 \pm 1.73) \%$, respectively. Statistical analysis showed that the percentage of both DCs and T-regs in model group decreased significantly compared to the control group $(P<0.05)$ (Figure 2$)$.

3.3. Colonic Mucosal Flt3 and Flt3L mRNA Expressions in Different Groups. In the model group, expressions of Flt3 and Flt3L mRNA in the colonic mucosa were significantly lower than those in the control group (Flt3: $0.53 \pm 0.06$ versus $1.01 \pm 0.13, P<0.05$; Flt3L: $0.18 \pm 0.10$ versus $1.01 \pm 0.25$, $P<0.05)$.

3.4. Protein Expressions of Flt3 in Colonic Mucosa and Flt3L in Plasma. Immunohistochemistry showed that Flt3 was expressed in colonic epithelial cells and colonic lymphocytes. Expression of Flt3 protein in the colonic mucosa in the model group was significantly lower than that in the control group $(31.66 \pm 2.31$ versus $82.19 \pm 5.34, P<0.05)$ (Figure 3$)$.

The results of ELISA showed that the level of Flt3L in plasma in the model group was significantly lower than that in the control group $(36.25 \pm 6.35$ versus $57.24 \pm 5.97, P<$ $0.05)$.

\section{Discussion}

$\mathrm{UC}$ is a major clinical syndrome of IBD and is characterized by chronic mucosal inflammation of the colon. The etiology and pathogenesis of UC are not completely clear.

The DSS-induced colitis model is widely used because of its simplicity and many similarities with human UC. DSS can induce an acute colitis characterized by bloody stools, ulcerations, and infiltration of inflammatory cells [10]. In our study, the results showed that DAI and histological scoring in the model group were significantly higher than those in the control group. Histologically, DSS produces submucosal 

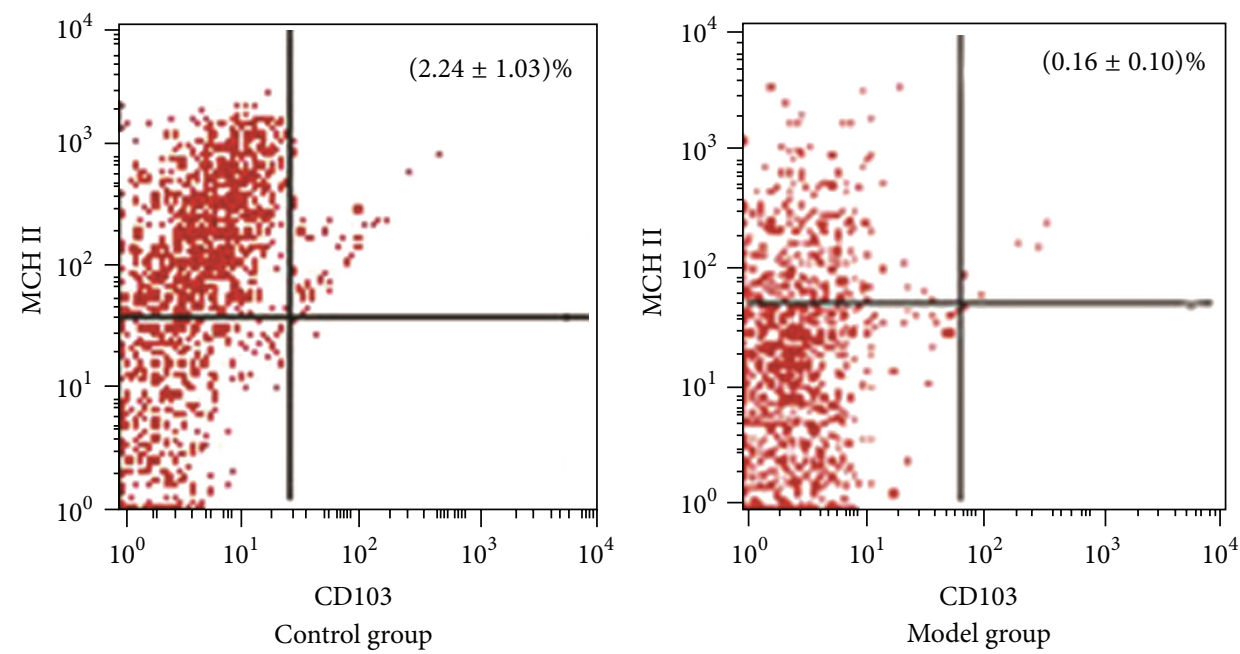

(a)
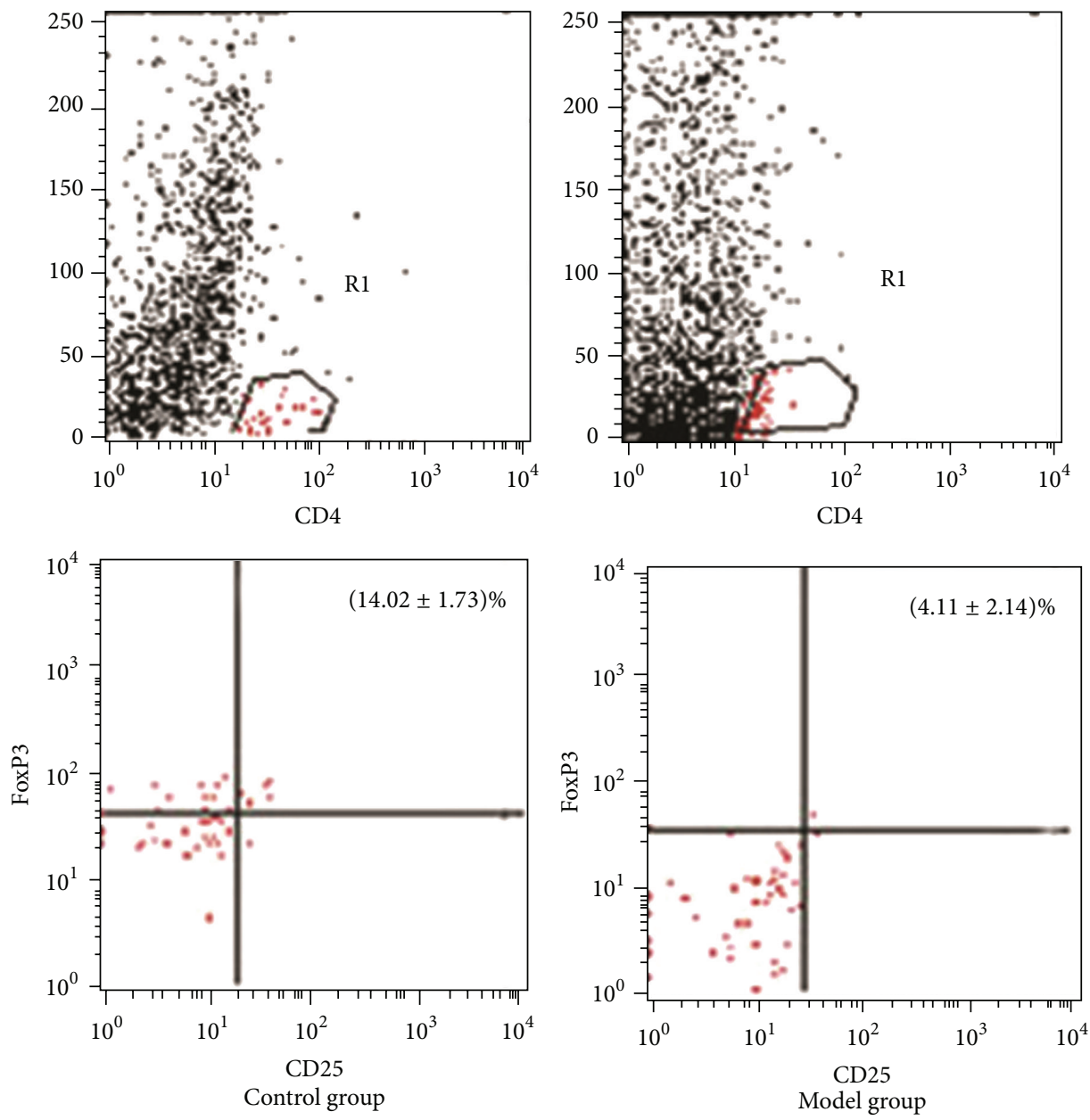

(b)

Figure 2: The percentage of $\mathrm{CD}_{103}{ }^{+} \mathrm{MHCII}^{+} \mathrm{DCs}$ and $\mathrm{CD} 4^{+} \mathrm{CD} 25^{+} \mathrm{FoxP}^{+} \mathrm{T}$-regs in the control and model group. (a) In the model group, the percentage of $\mathrm{CD} 03^{+} \mathrm{MHCII}^{+} \mathrm{DCs}$ in the total colonic mucosal cells was significantly lower than that in the control group $(0.16 \pm 0.10$ versus $2.24 \pm 1.03, P<0.05$ ). (b) Compared with control group, the percentage of $\mathrm{CD} 4^{+} \mathrm{CD} 25^{+}$FoxP $3^{+} \mathrm{T}$-regs in $\mathrm{CD} 4^{+} \mathrm{T}$ cells was significantly lower in the model group $(4.11 \pm 2.14$ versus $14.02 \pm 1.73, P<0.05)$. 

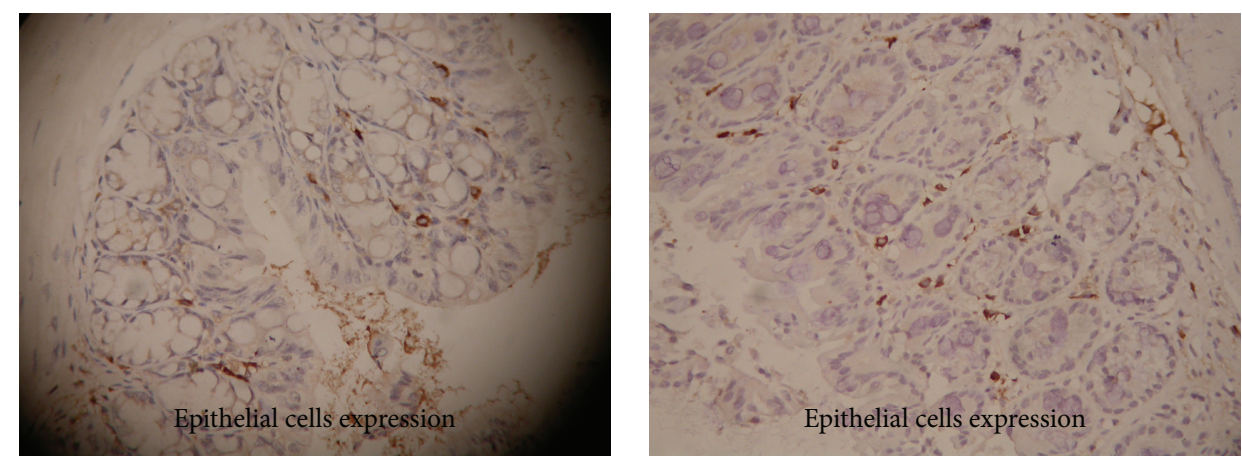

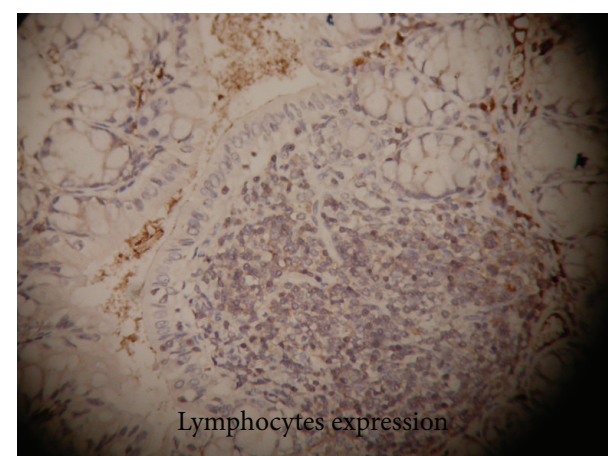

Control group

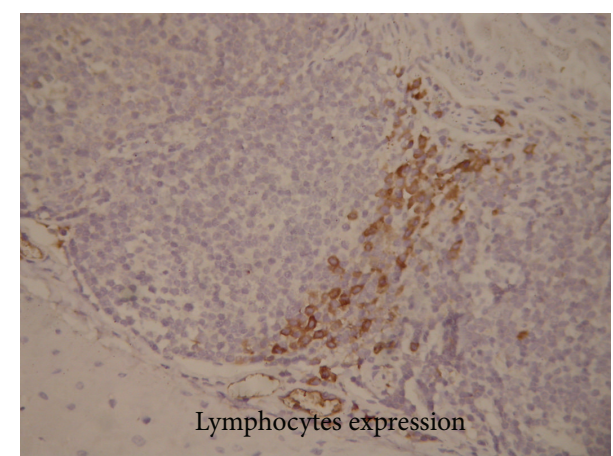

Model group

FIGURE 3: The colonic mucosal Flt3 expression in the control and UC model group. Immunohistochemistry showed that Flt3 was expressed in colonic epithelial cells and colonic lymphocytes. Expression of Flt3 protein in the colonic mucosa in the model group was significantly lower than that in the control group $(31.66 \pm 2.31$ versus $82.19 \pm 5.34, P<0.05)$.

erosions, ulceration, and inflammatory cell infiltration as well as crypt abscesses.

It is apparent that the perturbation of the delicate imbalance of gut immune response plays a relevant etiological role in UC. Gut immune responses are normally regulated to maintain a state of mucosal tolerance, which represents a balance of the need to increase the protective immunity toward pathogens while not activating damaging inflammatory responses to harmless luminal antigens [11].

Recent studies suggested that DCs and T-regs are involved in the maintenance of immunological tolerance in the gastrointestinal tract $[12,13]$. DCs, the most important antigen presenting cells (APC), are critically involved in the regulation of inflammatory response or immune tolerance, which mainly depends on different DCs subsets [14]. T-regs are a subpopulation of $\mathrm{T}$ cells including $\mathrm{CD} 4^{+} \mathrm{CD} 25^{+} \mathrm{FoxP} 3^{+}$ T-regs, Tr1, Th3, and $\mathrm{CD}^{+} \mathrm{T}$-regs, all of which serve to suppress the immune system and maintain self-tolerance.

The amount of $\mathrm{CD}_{103}{ }^{+} \mathrm{MHCII}^{+} \mathrm{DCs}$ and $\mathrm{CD} 4^{+} \mathrm{CD} 25^{+}$ FoxP $3^{+}$T-regs in colonic mucosa in terms of percentage was measured by FCM in our study. The results showed that the percentage of $\mathrm{CD} 103^{+} \mathrm{MHCII}^{+} \mathrm{DCs}$ and $\mathrm{CD} 4^{+} \mathrm{CD} 25^{+} \mathrm{FoxP}^{+}$T-regs in model group decreased significantly compared to the control group. This result indicated that $\mathrm{CD}_{103}{ }^{+} \mathrm{MHCII}^{+} \mathrm{DCs}$ and $\mathrm{CD}^{+} \mathrm{CD}^{+} 5^{+} \mathrm{FoxP}^{+} \mathrm{T}$-regs were involved in the imbalance of colonic mucosal immunology. A previous study has demonstrated that interactions between DCs and T cells seem to be essential for the development of immune tolerance, in which $\mathrm{CD} 4^{+} \mathrm{CD} 25^{+} \mathrm{FoxP}^{+} \mathrm{T}-$ regs are primed [5].

It was important for us to illustrate which pathway or factor was involved in regulating the balance between DCs and T-regs in the pathogenesis of UC. Latest study has found that there was a proposed homeostatic feedback loop between DCs and T-regs depending on Flt3/Flt3L; that is, increased numbers of DCs can result in an increased number of T-regs, and the increased T-regs can lead to a decrease in the number of DCs in a reverse way [15].

Flt3, a member of the tyrosine-kinase receptor family, was initially cloned from fetal liver cells with hematopoietic stem cell activity and was also expressed in DCs. Flt3L is the ligand for Flt3, which is a key regulatory cytokine for DCs commitment and development [16]. DCs, increased in number, can lead to expansion of T-regs by a mechanism of Flt3/Flt3L signal pathway that requires MHCII expression by DCs.

In this study, real-time PCR and immunohistochemistry were used to detect the Flt3 expression in colonic mucosa at the level of mRNA and protein. Our results indicated that expression of Flt 3 in the inflamed colonic mucosa was significantly lower than that in the controls. Mucosal Flt3L mRNA and plasma Flt3L protein were measured by realtime PCR as well as ELISA. The results showed that Flt3L was significantly lower in the model group than that in the 
control group. It implied that the insufficient expression of Flt3 and Flt3L was related to the development of UC. This is in accordance with the previous conclusion of Collins et al. [17].

As a matter of course, Flt3/Flt3L signaling is well known for its role in hematopoiesis and particularly in DC ontogeny and is thought to contribute predominantly to DC differentiation. The increase in T-regs induced by DC expansion is sufficient to prevent some autoimmune diseases such as type 1 autoimmune diabetes and IBD.

However, the specific function of mucosal DCs and the definite regulatory mechanism between DCs and T-regs are still unknown and need to be clarified in future work.

In conclusion, the reduction of Flt3 and Flt3L expression may possibly induce colonic immunoregulatory imbalance between $\mathrm{CD}_{103}{ }^{+} \mathrm{MHCII}^{+} \mathrm{DCs}$ and $\mathrm{CD}^{+} \mathrm{CD} 25^{+} \mathrm{FoxP}^{+}{ }^{+}$regs in DSS-induced colitis. Flt3/Flt3L participates in the process of regulating DCs and T-regs in the pathogenesis of UC, at least, in the acute stage of this disease.

\section{Conflict of Interests}

The authors declare that there is no conflict of interests regarding the publication of this paper.

\section{Acknowledgments}

The authors thank Yuejian Liu for his excellent technical assistance in helping with FCM. They also thank the SPF Laboratory Animal Center of Dalian Medical University.

\section{References}

[1] G. Bamias, G. Kaltsa, and S. D. Ladas, "Cytokines in the pathogenesis of ulcerative colitis," Discovery Medicine, vol. 11, no. 60, pp. 459-467, 2011.

[2] A. L. Hart, H. O. Al-Hassi, R. J. Rigby et al., "Characteristics of intestinal dendritic cells in inflammatory bowel diseases," Gastroenterology, vol. 129, no. 1, pp. 50-65, 2005.

[3] A. J. Macpherson and K. Smith, "Mesenteric lymph nodes at the center of immune anatomy," Journal of Experimental Medicine, vol. 203, no. 3, pp. 497-500, 2006.

[4] T. A. Kraus, L. Toy, L. Chan, J. Childs, and L. Mayer, "Failure to induce oral tolerance to a soluble protein in patients with inflammatory bowel disease," Gastroenterology, vol. 126, no. 7, pp. 1771-1778, 2004.

[5] K. Nagatani, K. Sagawa, Y. Komagata, and K. Yamamoto, "Peyer's patch dendritic cells capturing oral antigen interact with antigen-specific $\mathrm{T}$ cells and induce gut-homing $\mathrm{CD} 4{ }^{+} \mathrm{CD} 25$ + regulatory T cells in Peyer's patches," Annals of the New York Academy of Sciences, vol. 1029, pp. 366-370, 2004.

[6] A. Xiong, L. Duan, J. Chen et al., "Flt3L combined with rapamycin promotes cardiac allograft tolerance by inducing regulatory dendritic cells and allograft autophagy in mice," PLoS ONE, vol. 7, no. 10, Article ID e46230, 2012.

[7] K. S. Lee, N. Bosco, B. Malissen, R. Ceredig, and A. Rolink, "Expansion of peripheral naturally occurring T regulatory cells by Fms-like tyrosine kinase 3 ligand treatment," Blood, vol. 113, no. 25 , pp. $6277-6287,2009$.
[8] L. Stevceva, P. Pavli, G. Buffinton, A. Wozniak, and W. F. Doe, "Dextran sodium sulphate-induced colitis activity varies with mouse strain but develops in lipopolysaccharide-unresponsive mice," Journal of Gastroenterology and Hepatology (Australia), vol. 14, no. 1, pp. 54-60, 1999.

[9] L. A. Dieleman, M. J. H. J. Palmen, H. Akol et al., "Chronic experimental colitis induced by dextran sulphate sodium (DSS) is characterized by Th1 and Th2 cytokines," Clinical and Experimental Immunology, vol. 114, no. 3, pp. 385-391, 1998.

[10] S. Wirtz, C. Neufert, B. Weigmann, and M. F. Neurath, "Chemically induced mouse models of intestinal inflammation," Nature Protocols, vol. 2, no. 3, pp. 541-546, 2007.

[11] O. Annacker and F. Powrie, "Homeostasis of intestinal immune regulation," Microbes and Infection, vol. 4, no. 5, pp. 567-574, 2002.

[12] S. Makita, T. Kanai, Y. Nemoto et al., "Intestinal lamina propria retaining $\mathrm{CD} 4+\mathrm{CD} 25+$ regulatory $\mathrm{T}$ cells is a suppressive site of intestinal inflammation," Journal of Immunology, vol. 178, no. 8, pp. 4937-4946, 2007.

[13] D. C. Baumgart and S. R. Carding, "Inflammatory bowel disease: cause and immunobiology," The Lancet, vol. 369, no. 9573, pp. 1627-1640, 2007.

[14] D. C. Baumgart, D. Metzke, O. Guckelberger et al., "Aberrant plasmacytoid dendritic cell distribution and function in patients with Crohn's disease and ulcerative colitis," Clinical and Experimental Immunology, vol. 166, no. 1, pp. 46-54, 2011.

[15] G. Darrasse-Jèze, S. Deroubaix, H. Mouquet et al., "Feedback control of regulatory $\mathrm{T}$ cell homeostasis by dendritic cells in vivo," Journal of Experimental Medicine, vol. 206, no. 9, pp. 1853$1862,2009$.

[16] P. T. H. Coates, S. M. Barratt-Boyes, L. Zhang et al., "Dendritic cell subsets in blood and lymphoid tissue of rhesus monkeys and their mobilization with Flt3 ligand," Blood, vol. 102, no. 7, pp. 2513-2521, 2003.

[17] C. B. Collins, C. M. Aherne, E. N. McNamee et al., "Flt3 ligand expands $\mathrm{CD}_{103}{ }^{+}$dendritic cells and $\mathrm{FoxP}^{+}{ }^{+} \mathrm{T}$ regulatory cells, and attenuates Crohn's-like murine ileitis," Gut, vol. 61, no. 8, pp. 1154-1162, 2012. 


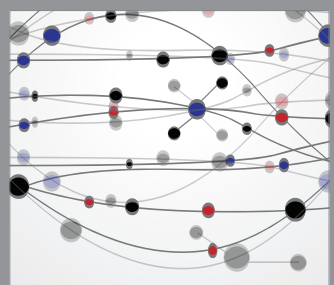

The Scientific World Journal
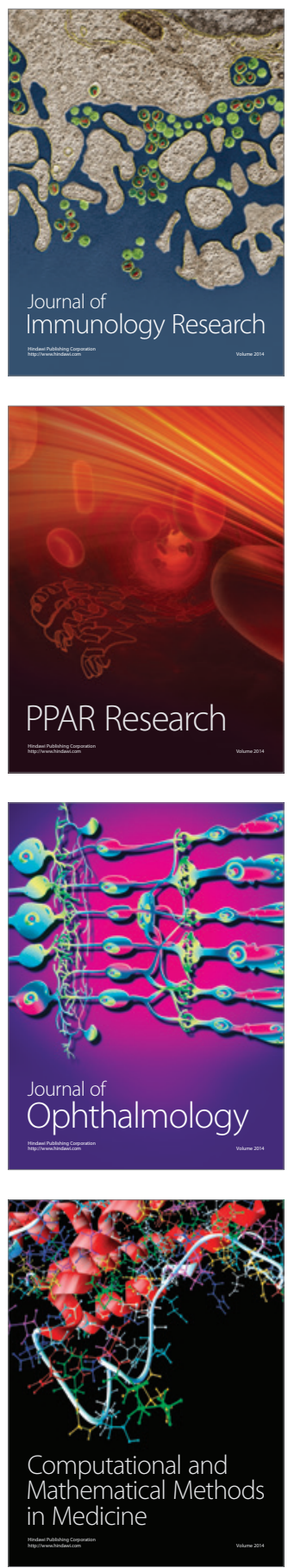

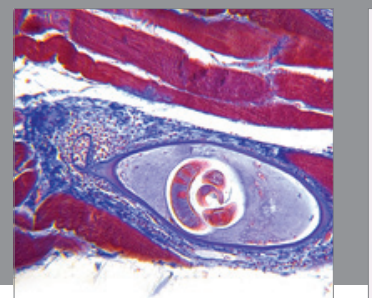

Gastroenterology

Research and Practice
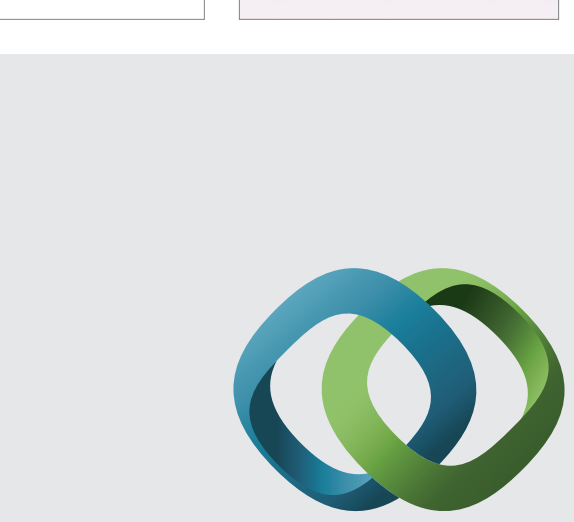

\section{Hindawi}

Submit your manuscripts at

http://www.hindawi.com
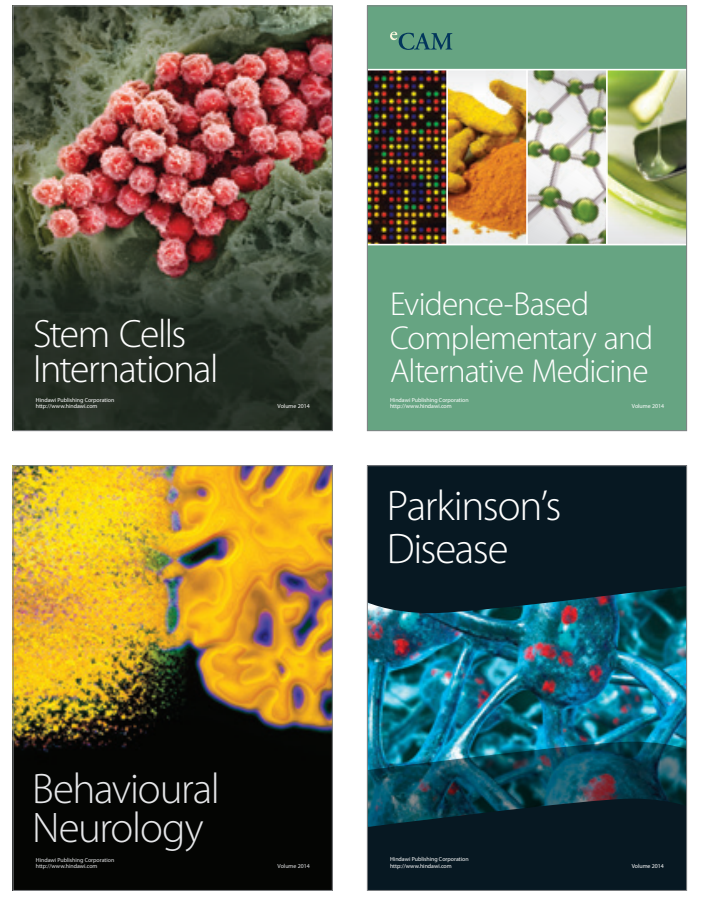
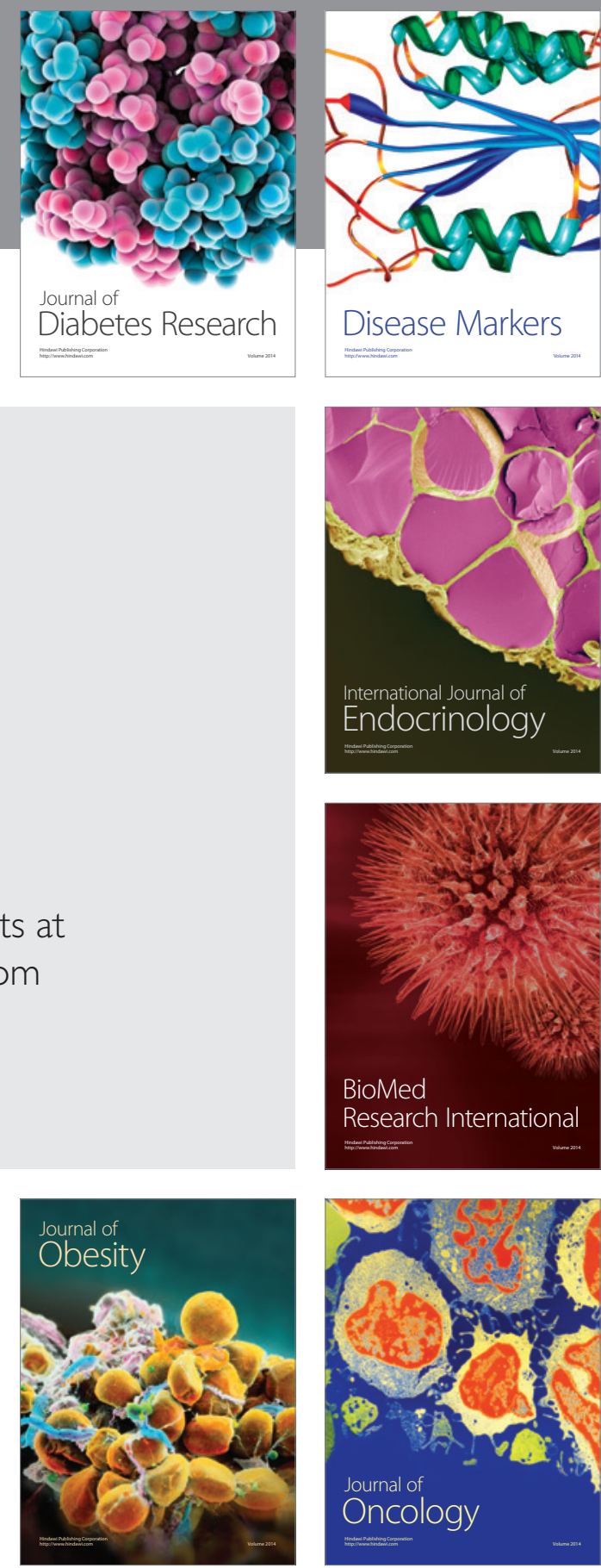

Disease Markers
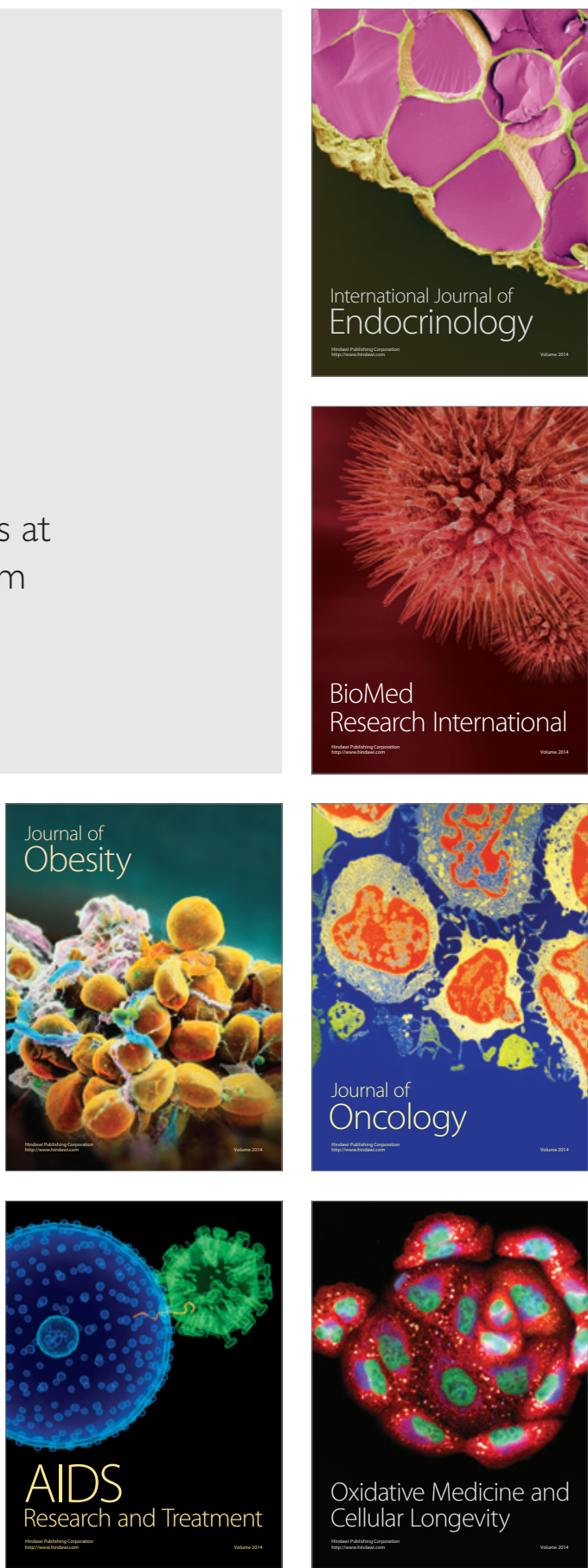